\title{
HEEGAARD GRADIENT OF SEIFERT FIBERED 3-MANIFOLDS
}

\author{
KAZUHIRO ICHIHARA
}

\begin{abstract}
The infimal Heegaard gradient of a 3-manifold was defined and studied by Marc Lackenby in an approach toward the well-known virtually Haken conjecture. As instructive examples, we consider Seifert fibered 3manifolds, and show that a compact orientable Seifert fibered 3-manifold has zero infimal Heegaard gradient if and only if it virtually fibers over the circle or over a surface other than the 2-sphere, equivalently, it has infinite fundamental group.
\end{abstract}

\section{INTRODUCTION}

The virtually Haken conjecture [3, Problem 3.2] is one of the most important conjectures in the study of 3-manifolds. Recently, in 4, Lackenby proved the following toward the virtually Haken conjecture. Let $M$ be a compact orientable 3manifold with boundary a (possibly empty) union of tori. If the fundamental group of $M$ fails to have Property $(\tau)$ with respect to some lattice of finite regular covers of $M$ and the lattice of covers has non-zero infimal (strong) Heegaard gradient, then $M$ is virtually Haken.

The infimal Heegaard gradient was introduced by Lackenby in [4 as follows. Recall that a Heegaard surface in a compact orientable 3-manifold means a closed embedded surface which separates the manifold into two compression bodies. It is well-known that every compact orientable 3-manifold contains a Heegaard surface. Let $\left\{M_{i} \rightarrow M\right\}$ be a collection of finite coverings of a compact orientable 3-manifold $M$ with covering degree $d_{i}$. Then the infimal Heegaard gradient of the collection $\left\{M_{i} \rightarrow M\right\}$ is defined as

$$
\inf _{i} \frac{\chi_{-}^{h}\left(M_{i}\right)}{d_{i}}
$$

where $\chi_{-}^{h}\left(M_{i}\right)$ denotes the negative of the maximal Euler characteristic of a Heegaard surface in $M_{i}$. The infimal Heegaard gradient of $M$ is defined as the infimal Heegaard gradient of the collection of all finite coverings of $M$. For brevity, we sometimes drop the word 'infimal'.

Toward the virtually Haken conjecture, on account of the Lackenby's result, our attention is attracted to the vanishing of Heegaard gradient. There actually exist 3manifolds with zero Heegaard gradient. Easy examples are given by surface bundles over the circle $S^{1}$, all of which are easily shown to have zero Heegaard gradient. In fact, Lackenby proposed a conjecture: a compact orientable hyperbolic 3-manifold has zero infimal Heegaard gradient if and only if it virtually fibers over the circle (Heegaard gradient conjecture 44). He showed in 4 this is also the case for closed

2000 Mathematics Subject Classification. 57M10, 57N10, 57M50.

Key words and phrases. Heegaard gradient, Seifert fibered 3-manifold.

The author is partially supported by JSPS Research Fellowships for Young Scientists. 
orientable reducible 3-manifolds, i.e., 3-manifolds which contain an embedded 2sphere not bounding a 3 -ball.

In order to analyze when Heegaard gradient vanishes, we consider Seifert fibered 3-manifolds as instructive examples and study which (covers of) Seifert fibered 3manifolds have zero Heegaard gradient. A Seifert fibered 3-manifold is defined as a 3-manifold with a decomposition into disjoint circles, called fibers, such that each circle has a neighborhood isomorphic to a fibered solid torus or Klein bottle. See [7] for the basic theory of Seifert fibered 3-manifolds. Then our main result is:

Theorem 1. A compact orientable Seifert fibered 3-manifold has zero infimal Heegaard gradient if and only if it virtually fibers over the circle or over a surface other than the 2-sphere. Equivalently, a compact orientable Seifert fibered 3-manifold has zero infimal Heegaard gradient if and only if its fundamental group is infinite.

Remark that there are infinitely many Seifert fibered 3-manifolds which admit a virtual fibration over a surface other than the 2 -sphere but do not admit that over the circle. In fact, it is known that such manifolds are precisely the 3-manifolds ad-

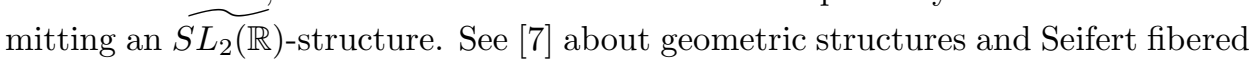
3 -manifolds for example.

To prove Theorem 1 we will actually construct a collection of finite covers with zero Heegaard gradient. Our advantage is using some circle bundles over surfaces as the finite covers, in which Heegaard surfaces are well understood. In addition, it will be also shown in Lemma 3 that all minimal genus Heegaard surfaces in these covers are strongly irreducible.

The strong irreducibility of Heegaard surface was introduced by Casson and Gordon in 2]. The Casson and Gordon's theorem is one of the excellent results about Haken manifolds and Heegaard splittings: if a compact orientable irreducible 3-manifold admits an irreducible but weakly reducible Heegaard surface, then the manifold is Haken. Here a Heegaard surface is called reducible if it has compressing disks on both sides whose boundaries coincide. A Heegaard surface that is not reducible is called irreducible. A Heegaard surface is called weakly reducible if it has compressing disks on both sides which are mutually disjoint. A Heegaard surface that is not weakly reducible is called strongly irreducible.

Motivated by this result, Lackenby also defined in [4] the infimal strong Heegaard gradient by considering only strongly irreducible Heegaard surfaces instead of all Heegaard surfaces. Precisely the infimal strong Heegaard gradient of a collection of finite coverings $\left\{M_{i} \rightarrow M\right\}$ of a compact orientable 3 -manifold $M$ with degree $d_{i}$ is defined as

$$
\liminf _{i} \frac{\chi_{-}^{s h}\left(M_{i}\right)}{d_{i}}
$$

where $\chi_{-}^{s h}\left(M_{i}\right)$ denotes the negative of the maximal Euler characteristic of a strongly irreducible Heegaard surface in $M_{i}$. Here we set $\chi_{-}^{s h}(M)=\infty$ if $M$ does not have such a Heegaard surface. In the same way as before, the infimal strong Heegaard gradient of $M$ is defined as the infimal strong Heegaard gradient of the collection of all finite coverings of $M$. Again, for brevity, we sometimes drop the word 'infimal'.

Toward the virtually Haken conjecture, we are also interested in the vanishing of strong Heegaard gradient. Concerning this, Lackenby also made a conjecture: every closed orientable hyperbolic 3-manifold has positive infimal strong Heegaard 
gradient (Strong Heegaard gradient conjecture [4]). In fact, he proved in [4 that a collection of the cyclic covers of a closed hyperbolic 3-manifold dual to a nontrivial element of $H_{2}(M)$ has non-zero strong Heegaard gradient. In particular, this implies that a collection of cyclic covers of a closed hyperbolic surface bundle over the circle which has zero Heegaard gradient has non-zero strong Heegaard gradient. On the contrary, we show that there are Seifert fibered 3-manifolds with zero infimal strong Heegaard gradient.

Proposition 1. A compact orientable Seifert fibered 3-manifold admitting an $\widetilde{S L_{2}(\mathbb{R}) \text { - }}$ structure has zero infimal strong Heegaard gradient.

We also consider the problem of which collections of finite coverings of a compact orientable Seifert fibered 3-manifold have zero Heegaard gradient. For a compact orientable hyperbolic 3-manifold $M$ of finite volume, Lackenby showed in 4 that a collection of the cyclic covers dual to a non-trivial element of $H_{2}(M, \partial M)$ has zero Heegaard gradient if and only if the manifold fibers over the circle and the element represents a fiber surface. Our result for Seifert fibered 3-manifolds is the following.

Theorem 2. Let $M$ be a closed orientable Seifert fibered 3-manifold with orientable base orbifold of negative Euler characteristic. Then the infimal Heegaard gradient of a collection $\left\{M_{i} \rightarrow M\right\}$ of finite coverings is zero if and only if the set of covering degrees between their regular fibers induced from $\left\{M_{i} \rightarrow M\right\}$ is unbounded.

Here each cover $M_{i}$ is endowed with a Seifert fibration induced from that of $M$ by covering projection. Note that the manifolds considered in Theorem 2 are precisely those admitting an $\widetilde{S L_{2}(\mathbb{R})}$-structure or an $\mathbb{H}^{2} \times \mathbb{R}$-structure. See [7] again.

We end this section with an easy observation that a compact orientable 3manifold has negative Heegaard gradient if and only if it is finitely covered by the 3 -sphere $S^{3}$. For let us consider the universal covering $\left\{S^{3} \rightarrow M\right\}$ with degree $d$, and then we have $\chi_{-}^{h}\left(S^{3}\right) / d=-2 / d<0$. Together with Theorem 1 this observation gives the following corollary.

Corollary 1. The Heegaard gradient of a Seifert fibered 3-manifold is always nonpositive.

Proof. By Theorem 1. Seifert fibered 3-manifolds with infinite fundamental group have zero Heegaard gradient. On the other hand, it is known that Seifert fibered 3manifolds with finite fundamental group is spherical, meaning that finitely covered by the 3 -sphere. Then they have negative Heegaard gradients as we observed above.

\section{Irreducible Heegaard surfaces in Seifert fibered 3-manifolds}

The crucial result we will use throughout this article is the classification of irreducible Heegaard surfaces of Seifert fibered 3-manifolds. It was proved by Moriah and Schultens in [5] that they are either vertical or horizontal.

We include here a brief treatment of these Heegaard surfaces. See [5, 8] for detailed descriptions. In this section, let $M$ be a compact orientable Seifert fibered 3-manifold. The base orbifold $O$ of $M$ is obtained by identifying each circle fiber to a point. A fiber of $M$ is called regular or exceptional if it corresponds to a regular point or a cone point of $O$ under the natural projection $M \rightarrow O$ respectively. 
First we give a construction of a vertical Heegaard surface in $M$. For simplicity we suppose that $M$ is closed and $O$ is orientable with at least two cone points. Take a minimal graph $\Gamma$ in the underlying surface $B$ of $O$ so that $B-\Gamma$ is a disk and $\Gamma$ is disjoint from the cone points of $O$. Choose a non-trivial partition of exceptional fibers of $M$. For the exceptional fibers in a half of the partition, add new edges to $\Gamma$ connecting $\Gamma$ and the cone points corresponding to the fibers. For all but one of the exceptional fibers in the other half, add new loops to $\Gamma$ encircling the cone points corresponding to the fibers. Lift the graph so obtained to $M$ by the natural projection. Add the exceptional fibers in the first half of the partition to the lifted graph in $M$, and take a regular neighborhood. It can be shown that its boundary surface becomes a Heegaard surface of $M$, which is called vertical. In the case where $M$ has less than two exceptional fibers or has non-empty boundary, similar construction can apply, and we have such a Heegaard surface called vertical. Thus all compact orientable Seifert fibered 3-manifold with orientable base orbifold contains a vertical Heegaard surface. Remark that the genus of a vertical Heegaard surface is independent from the partition chosen. It depends only upon the number of the exceptional fibers of $M$ (= the number of the cone points of $O$ ) and the genus of the underlying surface $B$ of $O$.

Next we consider a horizontal Heegaard surface in $M$. Unlike vertical ones, it only appears with two restrictions on $M$ as follows. Remove the interior of a fibered neighborhood of a regular or exceptional fiber from $M$. The complement, which remains a Seifert fibered 3-manifold, is known to be expressed as a surface bundle over the circle with a periodic monodromy. The first restriction on $M$ is that its fiber surface has a single boundary component. Recall that $M$ is reconstructed from the surface bundle by gluing the fibered neighborhood, which is a solid torus. Then the second restriction on $M$ is that the meridian of the fibered neighborhood intersects the boundary of a fiber surface exactly once. Under these restrictions, the boundary of the regular neighborhood of a fiber surface of the surface bundle yields a Heegaard surface in $M$, which is called horizontal.

The result obtained in 5 makes us possible to estimate the Heegaard genus, i.e., the minimal genus of a Heegaard surface, of a closed orientable Seifert fibered 3-manifold as follows.

Lemma 1. Let $M$ be a closed orientable Seifert fibered 3-manifold with orientable base orbifold. Then we have

$$
2 g(B)+k-2 \leq g(M) \leq 2 g(B)+k+1,
$$

where $g(M)$ denotes the Heegaard genus of $M, g(B)$ the genus of the underlying surface $B$ of the base orbifold of $M$ and $k$ the number of cone points of the base orbifold of $M$.

Proof. The upper bound is achieved by considering a vertical Heegaard surface. As stated above, such a Heegaard surface always exists and its genus is $2 g(B)+1$ if $k<2$ and $2 g(B)+k-1$ if $k \geq 2$. This was originally obtained in [1. Typically vertical Heegaard surfaces become minimal genus, but in some cases, horizontal Heegaard surfaces can be. Such cases were studied in [5], and it is shown in [8] that the Heegaard genus of the manifold is one less than that of a vertical Heegaard surface. This gives the lower bound of $g(M)$. 


\section{VANishing OF HEEgAARD GRADIENT}

In this section, we consider the vanishing of Heegaard gradient of Seifert fibered 3-manifolds and give a proof of Theorem [1] The next is the key proposition to prove the theorem.

Proposition 2. An orientable circle bundle over a closed orientable surface has zero Heegaard gradient if and only if its fundamental group is infinite.

Proof. Let $M$ be an orientable circle bundle over a closed orientable surface $F$ of genus $g$ and $b(M)$ the obstruction class of $M$, which is an integer-valued invariant. See 7 for the definition and the properties.

Assume that $M$ has finite fundamental group. Then $M$ is shown to be spherical, meaning that $M$ is finitely covered by the 3 -sphere. Then its Heegaard gradient is negative, in particular, is non-zero, as we observed in Section 1 Remark that $F$ is the 2-sphere and $b(M)$ is non-zero in this case.

Conversely assume that $M$ has infinite fundamental group. If $b(M)$ is zero, then $M$ is the trivial bundle $F \times S^{1}$, which has zero Heegaard gradient. Thus we assume that $b(M)$, denoted by $b$ simply, is non-zero. By taking the mirror image if necessary, we assume that $b>0$. Remark that the assumptions that $M$ has infinite fundamental group and $b>0$ imply that $F$ is not the 2 -sphere, i.e., $g \neq 0$.

Since $g$ is non-zero, we can take an $i$-fold covering $F_{i} \rightarrow F$ for $i \geq 2$. Note that the Euler characteristic $\chi\left(F_{i}\right)$ of $F_{i}$ is equal to $i(2-2 g)$, and so, its genus is $1-i(1-g)=i g-i+1$. This induces an $i$-fold covering $N_{i} \rightarrow M$, where $N_{i}$ is also a circle bundle over $F_{i}$. Note that the obstruction class of $N_{i}$ is equal to $b i$ [] Lemma 3.5].

Let $F_{i}^{0}$ be the compact surface obtained from $F_{i}$ by removing an open disk. Consider the manifold $F_{i}^{0} \times S^{1}$ and set the boundary of a surface fiber as a longitude and one of the circle fiber as a meridian on its boundary torus so that $N_{i}$ is regarded as the 3-manifold obtained from $F_{i}^{0} \times S^{1}$ by Dehn filling along the slope $b i$.

Consider the $b i$-fold covering $M_{i} \rightarrow N_{i}$ constructed in the following way. First we take the $b i$-fold cyclic covering of $F_{i}^{0} \times S^{1}$ in the $S^{1}$-direction. On the boundary of the cover, the preimage of the curve of slope $b i$ consists of a set of parallel curves of slope 1. Let $M_{i}$ be the Seifert fibered 3-manifold obtained from $F_{i}^{0} \times S^{1}$ by Dehn filling along this slope. Then it is easily seen that $M_{i}$ covers $N_{i}$ in the circle direction with degree $b i$.

The obstruction class of this $M_{i}$ is equal to $b i / b i=1$, and so, by [5] Corollary 0.5], the minimal genus Heegaard surface is horizontal. Then the Heegaard genus $g\left(M_{i}\right)$ of $M_{i}$ is equal to twice of the genus of $F_{i}$, that is, $2(i g-i+1)$. By construction, the covering $M_{i} \rightarrow M$ has degree $b i^{2}$, and so

$$
\inf _{i} \frac{\chi_{-}^{h}\left(M_{i}\right)}{b i^{2}}=\inf _{i} \frac{4(i g-i+1)-2}{b i^{2}} \leq \inf _{i} \frac{4 i g}{b i^{2}}=\inf _{i} \frac{4 g}{b i}=0 .
$$

Since $M$ has infinite fundamental group, each $\chi_{-}^{h}\left(M_{i}\right)$ is non-negative, and so, the collection $\left\{M_{i} \rightarrow M\right\}$ has zero Heegaard gradient. Also, since $M$ has infinite fundamental group, the collection of all finite coverings of $M$ has non-negative Heegaard gradient, and it must be zero as it includes $\left\{M_{i} \rightarrow M\right\}$. Thus we conclude that $M$ has zero Heegaard gradient.

Lemma 2. Let $M$ be a compact orientable 3-manifold and $\widetilde{M}$ a finite cover of $M$. If $\widetilde{M}$ has zero Heegaard gradient, then also $M$ has. 
Proof. Assume that $\widetilde{M}$ has zero Heegaard gradient. Note that $M$, hence $\widetilde{M}$, is not finitely covered by the 3 -sphere. For, if $M$ were, then $M$ and $\widetilde{M}$ must have negative Heegaard gradient.

Let $d$ be the covering degree of $\widetilde{M} \rightarrow M$ and $\left\{\widetilde{M}_{i} \rightarrow \widetilde{M}\right\}$ the collection of all finite covering of $\widetilde{M}$ with degree $\widetilde{d}_{i}$. The set of compositions $\left\{\widetilde{M}_{i} \rightarrow M\right\}$ gives a collection of finite covering of $M$ with degree $\widetilde{d}_{i} d$. Since $M$ is not finitely covered by the 3 -sphere, any $\chi_{-}^{h}\left(\widetilde{M}_{i}\right)$ is non-negative. Thus we have

$$
0 \leq \inf _{i} \frac{\chi_{-}^{h}\left(\widetilde{M}_{i}\right)}{\widetilde{d}_{i} d} \leq \inf _{i} \frac{\chi_{-}^{h}\left(\widetilde{M}_{i}\right)}{\widetilde{d}_{i}}
$$

Now the right term becomes zero, and so $\left\{\widetilde{M}_{i} \rightarrow M\right\}$ has zero Heegaard gradient. This implies that the Heegaard gradient of the set of all finite coverings of $M$ is non-positive. But it must be zero as $M$ is not finitely covered by the 3 -sphere. Therefore $M$ has zero Heegaard gradient.

Proof of Theorem 1. Let $M$ be a compact orientable Seifert fibered 3-manifold, $e(M)$ the Euler number of $M, O$ the base orbifold of $M$ and $\chi(O)$ the Euler characteristic of $O$.

First suppose that $M$ does not virtually fiber over the circle nor over a surface other than the 2-sphere. Then it is known that $M$ is spherical, equivalently the fundamental group $\pi_{1}(M)$ of $M$ is finite. As we remarked before, it has negative Heegaard gradient, in particular, its Heegaard gradient cannot vanish. Note that $e(M) \neq 0$ and $\chi(O)>0$ hold in this case.

Assume conversely that $M$ virtually fibers over the circle or over a surface other than the 2-sphere. Note that this is equivalent to that $M$ has infinite fundamental group. In this case, $e(M)=0$ or $\chi(O) \leq 0$ hold.

If $M$ virtually fibers over the circle, equivalently $e(M)$ is zero, then it has zero Heegaard gradient as stated in Section 1. Remark that if $M$ has non-empty boundary, then $e(M)$ is always zero.

The remaining case is that $M$ virtually fibers over a surface $F$ other than the 2-sphere. Since $\chi(O) \leq 0, F$ has non-positive Euler characteristic, and so, a finite cover $\widetilde{M}$ of $M$ fibering over $F$ must have infinite fundamental group. Taking a double cover if necessary, we can assume that $F$ is orientable. Then, by Proposition 2. $\widetilde{M}$ has zero Heegaard gradient. This completes the proof of the theorem together with Lemma 2

In the proof, remark that if $e(M) \neq 0$ and $\chi(O)=0$, then $M$ is a Nil-manifold, each of which is known to be a virtual torus bundle over the circle. Precisely, a closed Seifert fibered 3-manifold with infinite fundamental group does not virtually fiber over the circle if and only if it admits a geometric structure modeled on $\widetilde{S L_{2} \mathbb{R}}$. See [7] for example.

\section{3-MANifolds With Zero Strong HEegaARd GRAdiEnt}

Here we consider an orientable circle bundle over a closed orientable surface other than the 2-sphere. It is shown to be irreducible and to contain essential tori. Thus it is a Haken manifold. 
Lemma 3. Let $M$ be an orientable circle bundle over a closed orientable surface with the obstruction class \pm 1 . Then every irreducible Heegaard surface in $M$ is strongly irreducible.

Equivalently such a 3-manifold $M$ contains no irreducible but weakly reducible Heegaard surface. Thus $M$ is not recognized to be Haken by using the Casson and Gordon's theorem.

Proof of Lemma 3. Since the Euler number of $M$ is equal to $\pm 1, M$ has a unique (up to homeomorphism) irreducible Heegaard surface $S$ which is horizontal 5 , Corollary $0.5]$. Note that the genus of $S$ is just twice of that of the base surface $F$ of $M$. Moreover, Corollary 2 in [6] says that if $S$ is weakly reducible, then it is also vertical. In our case, a vertical Heegaard surface has genus at least twice of that of $F$, and so, $S$ must be strongly irreducible.

Proof of Proposition 1 Let $M$ be a compact orientable Seifert fibered 3-manifold which admits an $\widetilde{S L_{2}(\mathbb{R})}$-structure. It is known that $M$ is finitely covered by an orientable circle bundle over a closed orientable surface other than the 2-sphere with non-zero obstruction class. Then, as in the proofs of Theorem 1 and Lemma 2] we can find a collection of finite covers $\left\{M_{i} \rightarrow M\right\}$ of degree $d_{i}$ such that

$$
\inf _{i} \frac{\chi_{-}^{h}\left(M_{i}\right)}{d_{i}}=0 .
$$

Moreover, by the proof of Proposition 2 we can take an orientable circle bundle over a closed orientable surface with obstruction class \pm 1 as each $M_{i}$. Then we have $\chi_{-}^{h}\left(M_{i}\right)=\chi_{-}^{s h}\left(M_{i}\right)$ holds. For Lemma 3 assures that $\chi_{-}^{h}\left(M_{i}\right)$ is attained by a strongly irreducible Heegaard surface. It implies that the collection of finite covers $\left\{M_{i} \rightarrow M\right\}$ has zero strong Heegaard gradient. Thus the strong Heegaard gradient of $M$ is non-positive. In fact, it must be zero because it is at least Heegaard gradient of $M$ which is zero as we proved in Theorem 1

\section{VANishing of HeEgaARD gradient For Finite COVERS}

In this section, we consider when a collection of finite coverings of a Seifert fibered 3-manifold has zero Heegaard gradient and give a proof of Theorem 2

Lemma 4. Let $M$ be a closed orientable irreducible Seifert fibered 3-manifold with infinite fundamental group and orientable base orbifold, and $\left\{M_{i} \rightarrow M\right\}$ a collection of finite coverings with degree $d_{i}$. Then the Heegaard gradient of $\left\{M_{i} \rightarrow M\right\}$ is zero if and only if $\inf _{i} g\left(M_{i}\right) / d_{i}$ is zero, where $g\left(M_{i}\right)$ denotes the Heegaard genus of $M_{i}$.

Proof. Assume that $\inf _{i} g\left(M_{i}\right) / d_{i}=0$ holds. Then

$$
0 \leq \inf _{i} \frac{\chi_{-}^{h}\left(M_{i}\right)}{d_{i}}=\inf _{i} \frac{2 g\left(M_{i}\right)-2}{d_{i}} \leq \inf _{i} \frac{2 g\left(M_{i}\right)}{d_{i}}=0 .
$$

Here the first inequality follows from the fact that $M$ has infinite fundamental group. Thus we have the Heegaard gradient of $\left\{M_{i} \rightarrow M\right\}$ is zero.

Conversely assume that the Heegaard gradient of $\left\{M_{i} \rightarrow M\right\}$ is zero. We remark that $\chi_{-}^{h}\left(M_{i}\right)>0$ holds for every $M_{i}$. Otherwise the Heegaard genus $g\left(M_{i}\right)$ is at most 1 , contradicting the fact that $M$ is irreducible and $\pi_{1}(M)$ is infinite. It 
follows that $\left\{M_{i} \rightarrow M\right\}$ is an infinite collection and the set $\left\{d_{i}\right\}$ an unbounded set of positive integers. Then we have

$$
0 \leq \inf _{i} \frac{g\left(M_{i}\right)}{d_{i}} \leq \inf _{i} \frac{2 g\left(M_{i}\right)}{d_{i}} \leq \inf _{i}\left(\frac{\chi_{-}^{h}\left(M_{i}\right)}{d_{i}}+\frac{2}{d_{i}}\right) .
$$

By the assumption that the Heegaard gradient of $\left\{M_{i} \rightarrow M\right\}$ is zero, we can find a subsequence $\left\{M_{j} \rightarrow M\right\}$ such that

$$
\frac{\chi_{-}^{h}\left(M_{j}\right)}{d_{j}} \rightarrow 0 \quad \text { as } \quad j \rightarrow \infty .
$$

Since $\chi_{-}^{h}\left(M_{i}\right)>0$ holds for every $M_{i}$, we have $d_{j} \rightarrow \infty$ as $j \rightarrow \infty$. Thus we conclude

$$
\left(\frac{\chi_{-}^{h}\left(M_{j}\right)}{d_{j}}+\frac{2}{d_{j}}\right) \rightarrow 0 \quad \text { as } \quad j \rightarrow \infty
$$

This indicates that

$$
\inf _{i}\left(\frac{\chi_{-}^{h}\left(M_{i}\right)}{d_{i}}+\frac{2}{d_{i}}\right)=0,
$$

and the proof is completed.

Proof of Theorem 2. We first remark that $M$ is irreducible and has infinite fundamental group. This follows from the assumption that the base orbifold of $M$ has negative Euler characteristic. See [7] for an example.

Let $m_{i}$ be the covering degree between the regular fibers of $M_{i}$ and $M$ induced from $\left\{M_{i} \rightarrow M\right\}$. Also let $l_{i}$ be the degree of the orbifold-covering between the base orbifolds $O_{i}$ and $O$ of $M_{i}$ and $M$ induced from $\left\{M_{i} \rightarrow M\right\}$. Note that $d_{i}=m_{i} l_{i}$ holds.

First assume that the Heegaard gradient of $\left\{M_{i} \rightarrow M\right\}$ is zero. By Lemma 4 this implies $\inf _{i} g\left(M_{i}\right) / d_{i}=0$ holds, where $g\left(M_{i}\right)$ denotes the Heegaard genus of $M_{i}$ and $d_{i}$ the covering degree of $M_{i} \rightarrow M$. Also by Lemma 1 we have

$$
g\left(M_{i}\right) \geq 2 g\left(B_{i}\right)+k_{i}-2,
$$

where $g\left(B_{i}\right)$ denotes the genus of the underlying surface $B_{i}$ of $O_{i}$ and $k_{i}$ the number of cone points of $O_{i}$. Consider the Euler characteristic $\chi\left(O_{i}\right)$ of $O_{i}$. This is calculated as

$$
\chi\left(O_{i}\right)=2-2 g\left(B_{i}\right)-\sum_{j=1}^{k_{i}}\left(1-\frac{1}{\alpha_{i j}}\right),
$$

where $\alpha_{i j}$ denotes the index of the $j$-th cone point of $O_{i}$. Then we have

$$
2 g\left(B_{i}\right)+k_{i}-2=-\chi\left(O_{i}\right)+\sum_{j=1}^{k_{i}} \frac{1}{\alpha_{i j}} \geq-\chi\left(O_{i}\right) .
$$

This implies that

$$
g\left(M_{i}\right) \geq 2 g\left(B_{i}\right)+k_{i}-2 \geq-\chi\left(O_{i}\right)=-l_{i} \chi(O),
$$

where $\chi(O)$ denotes the Euler characteristic of $O$. Consequently we obtain

$$
0=\inf _{i} \frac{g\left(M_{i}\right)}{d_{i}} \geq \inf _{i} \frac{-l_{i} \chi(O)}{m_{i} l_{i}}=\inf _{i} \frac{-\chi(O)}{m_{i}} .
$$

By assumption, $\chi(O)$ is negative, and hence, the set $\left\{m_{i}\right\}$ must be unbounded. 
Next we conversely assume that $\left\{m_{i}\right\}$ is unbounded. By Lemma 1 we have

$$
g\left(M_{i}\right) \leq 2 g\left(B_{i}\right)+k_{i}+1 .
$$

In the same way as above,

$$
2 g\left(B_{i}\right)+k_{i}+1=-\chi\left(O_{i}\right)+3+\sum_{j=1}^{k_{i}} \frac{1}{\alpha_{i j}}
$$

holds. With $\alpha_{i j} \geq 2$, we obtain

$$
g\left(M_{i}\right) \leq-\chi\left(O_{i}\right)+3+\frac{k_{i}}{2} .
$$

Let $k$ be the number of cone points of $O$. Then $k_{i}$ is at most $l_{i} k$. Thus we conclude

$$
\begin{aligned}
0 & \leq \inf _{i} \frac{g\left(M_{i}\right)}{d_{i}} \leq \inf _{i} \frac{-\chi\left(O_{i}\right)+3+k_{i} / 2}{d_{i}} \\
& \leq \inf _{i} \frac{-l_{i} \chi(O)+3+l_{i} k / 2}{m_{i} l_{i}} \leq \inf _{i}\left(\frac{-\chi(O)+k / 2}{m_{i}}+\frac{3}{m_{i} l_{i}}\right) .
\end{aligned}
$$

By the assumption that $\left\{m_{i}\right\}$ is unbounded, we can find a subsequence $\left\{M_{j} \rightarrow M\right\}$ such that $m_{j} \rightarrow \infty$ as $j \rightarrow \infty$. Then, since $l_{i} \geq 1$ for any $i$,

$$
\left(\frac{-\chi(O)+k / 2}{m_{j}}+\frac{3}{m_{j} l_{j}}\right) \rightarrow 0 \quad \text { as } \quad j \rightarrow \infty
$$

holds. Together with the inequality above, we obtain $\inf _{i} g\left(M_{i}\right) / d_{i}=0$. By Lemma [4. this is equivalent to that the Heegaard gradient of $\left\{M_{i} \rightarrow M\right\}$ is zero.

\section{ACKNowledgements}

The author would like to thank Yo'av Rieck for helpful discussions. He also thanks to Marc Lackenby and Tsuyoshi Kobayashi for helpful comments and to Eric Sedgwick for suggestions about Proposition 1

\section{REFERENCES}

[1] M. Boileau and H. Zieschang, 'Heegaard genus of closed orientable Seifert 3-manifolds', Invent. Math. 76 (1984) 455-468.

[2] A. J. Casson and C. McA. Gordon, 'Reducing Heegaard splittings', Topology Appl. 27 (1987) $275-283$.

[3] R. Kirby, 'Problems in low-dimensional topology', Geometric topology, AMS/IP Stud. Adv. Math., 2.2, (Athens, GA, 1993), (Amer. Math. Soc., Providence, RI, 1997) pp.35-473.

[4] M. Lackenby, 'Heegaard splittings, the virtually Haken conjecture and Property $(\tau)$ ', preprint, math.GT/0205327

[5] Y. Moriah and J. Schultens, 'Irreducible Heegaard splittings of Seifert fibered spaces are either vertical or horizontal', Topology 37 (1998) 1089-1112.

[6] J. Schultens, 'Weakly reducible Heegaard splittings of Seifert fibered spaces', Topology Appl. 100 (2000) 219-222.

[7] P. Scott, 'The geometries of 3-manifolds', Bull. London Math. Soc. 15 (1983) 401-487.

[8] E. Sedgwick, 'The irreducibility of Heegaard splittings of Seifert fibered spaces', Pacific J. Math. 190 (1999) 173-199.

Department of Information and Computer Sciences, Nara Women's University, KitaUOYA Nishimachi, NARA 630-8506

E-mail address: ichihara@vivaldi.ics.nara-wu.ac.jp 